\title{
Synthesis of the polymerizable room temperature ionic liquid AMPS - TEA and superabsorbency for organic liquids of its copolymeric gels with acrylamide
}

\author{
Tingting Weng ${ }^{\mathrm{a}}$, Jianwei Guo ${ }^{\mathrm{a}}$, Xinming $\mathrm{Li}^{\mathrm{b} *}$, Yingde Cui ${ }^{\mathrm{a}, \mathrm{b}}$, Xuejiao Yang ${ }^{\mathrm{a}}$, Kun Zhang ${ }^{\mathrm{a}}$, Buning Zhang ${ }^{\mathrm{b}}$, \\ Guoqiang Yin ${ }^{\mathrm{b}}$, Sergey V. Mikhalovsky, ${ }^{\mathrm{c}, \mathrm{d}}$, Lyuba I. Mikhalovska ${ }^{\mathrm{c}}$, Irina N. Savina ${ }^{\mathrm{c}}$, Carol A. Howel ${ }^{\mathrm{c}}$ and \\ Susan R. Sandeman ${ }^{\mathrm{c}}$ \\ ${ }^{a}$ School of Chemical Engineering and Light Industry, Guangdong University of Technology, Guangzhou 510006, China; ${ }^{b}$ School of \\ Chemistry and Chemical Engineering, Zhongkai University of Agriculture and Engineering, No. 24 Dongsha Street, Haizhu District, \\ Guangzhou 510225, P.R. China; 'School of Pharmacy and Biomolecular Science, University of Brighton, Lewes Road, BN2 4GJ, \\ Brighton, UK; ${ }^{d}$ School of Engineering, Nazarbayev University, Kabanbay batyr Avenue, Astana 010000, Kazakhstan
}

(Received 23 May 2013; accepted 14 June 2013)

\begin{abstract}
A polymerizable room temperature ionic liquid (RTIL), 2-acrylamido-2-methyl-1-propane sulfonic acid (AMPS) triethylamine (TEA), was synthesized by neutralization of AMPS with TEA in acetone followed by evaporation of the solvent under a reduced pressure at room temperature. The RTIL was characterized with fourier transform infrared spectroscopy, differential scanning calorimetry (DSC), and ${ }^{1} \mathrm{H}$ NMR. Co-polymeric gels of the RTIL with acrylamide (AAm) were prepared by aqueous solution polymerization using $\mathrm{N}, \mathrm{N}^{\prime}$-methylenebisacrylamide as a crosslinker, and ammonium persulfate as an initiator. Superabsorbency of the gels in aqueous and a series of organic liquids was investigated gravimetrically. DSC data showed that the glass transition temperature of AMPS - TEA was $-59.4{ }^{\circ} \mathrm{C}$. Poly (AMPS - TEA-co-AAm) gels exhibited superabsorbency in both water and a series of organic solvents. The mechanism for swelling in aqueous and organic media of the gels was critically discussed.
\end{abstract}

Keywords: 2-acrylamido-2-methyl-1-propane sulfonic acid (AMPS); triethylamine (TEA); room temperature ionic liquid (RTIL); acrylamide; superabsorbency

\section{Introduction}

Being melting salts at room temperature and completely composed of discrete cations and anions, room temperature ionic liquids (RTILs) have received much interest due to their unique properties such as negligible vapor pressure, thermal stability, chemical stability and nonflammability, relatively high ionic conductivity and wide potential window.[1-13] Based on such properties, a large amount of applications, such as solvent in synthesis, catalysis, biocatalysis and in electrochemistry, have been explored.[14-18] Recently, using of RTILs to form polymeric materials was found to take an enabling role in some fields of polymer chemistry and material science.[19-23] This includes immobilizing RTILs in solid devices and polymerizing RTILs with unsaturated bonds to give a special type of polyelectrolytes that carry an RTIL species in each of the repeating units.[24-34] In this work, a polymerizable RTIL, 2-acrylamido-2-methyl-1-propane sulfonic acid (AMPS), and triethylamine (TEA) was synthesized by neutralization of AMPS and TEA, the co-polymeric gels of the RTIL with acrylamide (AAm) was synthesized, and it was found that the gels showed superabsorbency for both water and a variety of organic solvents. The molecular structures of the chemicals used in this work are shown in Scheme 1.

\section{Experimental}

\section{Materials}

AMPS, Sigma-Aldrich; AAm, N,N'-methylenebisacrylamide (MBAm), ammonium persulfate (APS), TEA, acetone, they were analytical reagent grade and used as received without further purification.

\section{Synthesis of AMPS - TEA}

About $20 \mathrm{~g}$ acetone was added to a flask, then added $5 \mathrm{~g}$ AMPS, and $2.50 \mathrm{~g}$ TEA was added to the flask in drops under stirring. When the powder in the solution disappeared and the solution turned clear, stopped stirring and evaporated the solvent under a reduced pressure at room temperature, to give a transparent and pale yellow liquid.

\footnotetext{
*Corresponding author. Email: lixinming@sina.com 


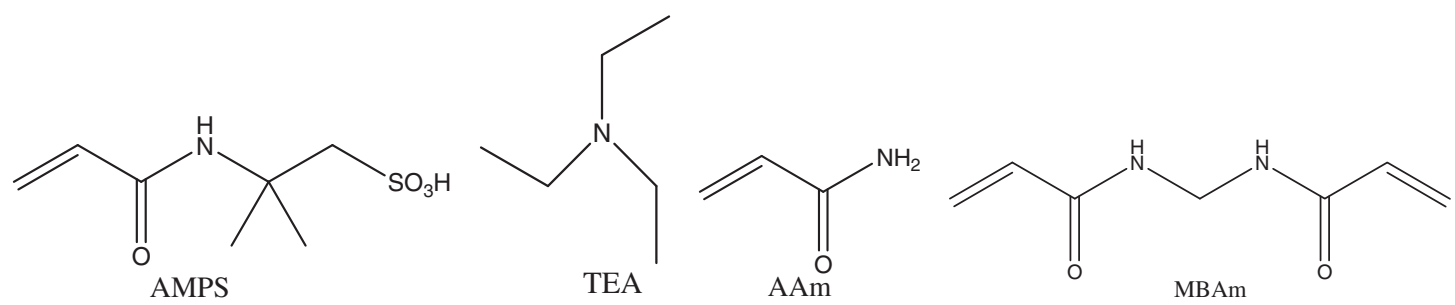

Scheme 1. Structure of the chemicals to synthesize poly(AMPS - TEA-co-AAm) gels.

\section{Characterizations of AMPS - TEA}

Differential scanning calorimetry (DSC) analysis was preformed for AMPS - TEA with Mettler Teledo DSC1. The measurement conditions were with a scanning rate of $10{ }^{\circ} \mathrm{C} / \mathrm{min}$ and nitrogen gas flow rate of $50 \mathrm{ml} / \mathrm{min}$. Fourier transform infrared spectroscopy (FT-IR) spectrophotometer (Bruker Vector) was used to characterize AMPS - TEA as well. ${ }^{1} \mathrm{H}$ NMR (nuclear magnetic resonance) characterization for AMPS - TEA was performed with Bruker AV400 using $\mathrm{CDCl}_{3}$ as a solvent.

\section{Synthesis of poly(AMPS - TEA-co-AAm) gels}

AMPS - TEA, AAm, MBAm, and APS were dissolved in water successively to give reaction solution. The dosage of APS was $0.1 \%$ gross weight of the reaction solution. The total monomers concentration of the reaction solution was $40 \%$ by weight. The dosage of MBAm and the proporation of AMPS - TEA (calculated by AMPS) and AAm varied to give hydrogels with different compositions.

The reaction solution was heated in a water bath with $50^{\circ} \mathrm{C}$ for $24 \mathrm{~h}$. The resulted hydrogels was immersed in distilled water for 7 days and the water was changed every $24 \mathrm{~h}$ to remove water-soluble materials, followed by drying the hydrogels in an oven at $105^{\circ} \mathrm{C}$ to give xerogel.

\section{Swelling measurements of poly(AMPS - TEA-co-AAm) gels}

The xerogel of poly(AMPS - TEA-co-AAm) was immersed in water and a variety of organic solvents to test the swelling characteristics. The equilibrium swelling behavior of the samples was measured by a gravimetric method. The samples were made to swell with distilled water and various organic solvents at room temperature until the swelling equilibrium were reached, followed by being removed and blotted with filter paper to remove the overloaded liquids on the surface, and weighed. The equilibrium swelling ratio was defined as follows:

$$
\text { Equilibrium swelling ratio }(Q, \mathrm{~g} / \mathrm{g})=\frac{W_{\mathrm{w}}-W_{\mathrm{d}}}{W_{\mathrm{d}}}
$$

where $W_{\mathrm{w}}$ was weight of the swollen gels and $W_{\mathrm{d}}$ was weight of the xerogel.

\section{Results and discussion}

\section{Synthesis of AMPS - TEA}

In the present work, AMPS - TEA was prepared by neutralizing AMPS with TEA in acetone followed by evaporation of the solvent under a reduced pressure at room temperature; the reaction can be expressed by Scheme 2. It was found that the resulted salt was transparent liquids with pale yellow at room temperature, DSC showed that the glass transition temperature was about $-59.4^{\circ} \mathrm{C}$, as shown in Figure 1 .

\section{Characterization of AMPS - TEA}

The resulted salt was characterized with FT-IR and ${ }^{1} \mathrm{H}$ NMR. The FT-IR spectrum and the ${ }^{1} \mathrm{H}$ NMR spectrum of the salt were shown in Figures 2 and 3, respectively. In Figure 2, characteristic peaks of $-\mathrm{SO}_{3}$ were shown. Peak $620 \mathrm{~cm}^{-1}$ is absorption of the $\mathrm{C}-\mathrm{S}$ bond, peak $1040 \mathrm{~cm}^{-1}$ is absorption of the $\mathrm{S}-\mathrm{O}$ bond, and peak $1200 \mathrm{~cm}^{-1}$ was the absorption of $-\mathrm{S}=\mathrm{O}$. Other characteristic peaks of the salt are also shown. Peak $1640 \mathrm{~cm}^{-1}$ is absorption of the $-\mathrm{C}=\mathrm{C}$ bond, and peak $1560 \mathrm{~cm}^{-1}$ is absorption of the $-\mathrm{C}-\mathrm{N}$ bond. Combining with the values of chemical shift of the resulted salt shown in Figure 3, the structure of the resulted salt can be decided, as shown in Figure 4.

\section{Synthesis of poly(AMPS - TEA-co-AAm) gels}

Poly(AMPS - TEA-co-AAm) gels were prepared by free radical aqueous solution co-polymerization of AMPS - TEA, MBAm, and AAm. The resulted poly (AMPS - TEA-co-AAm) was transparent and glassy hydrogels. The formation of the crosslinked networks can be expressed by Scheme 3 . 


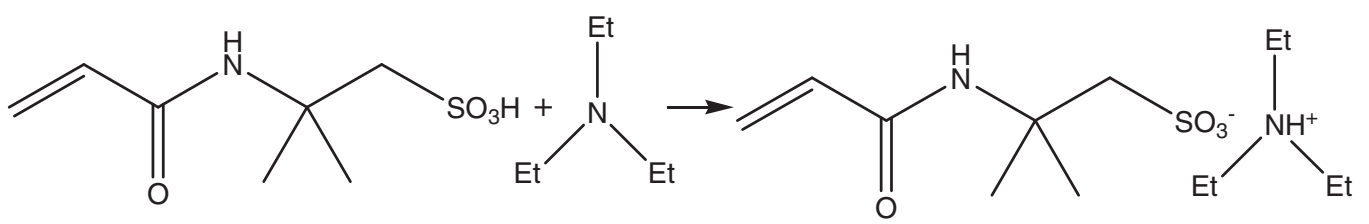

Scheme 2. Preparation of AMPS - TEA.

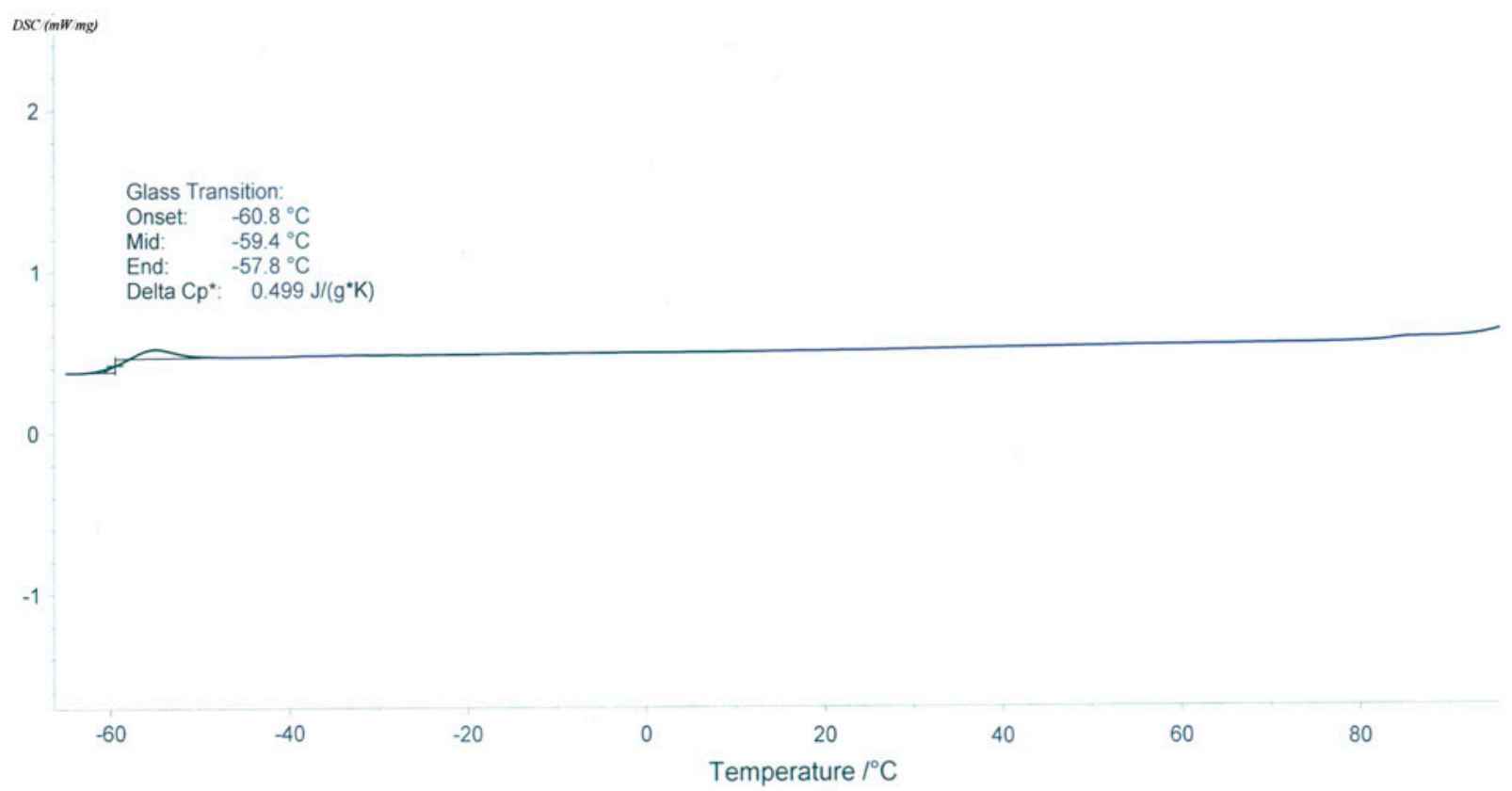

Figure 1. DSC characterization of AMPS - TEA.

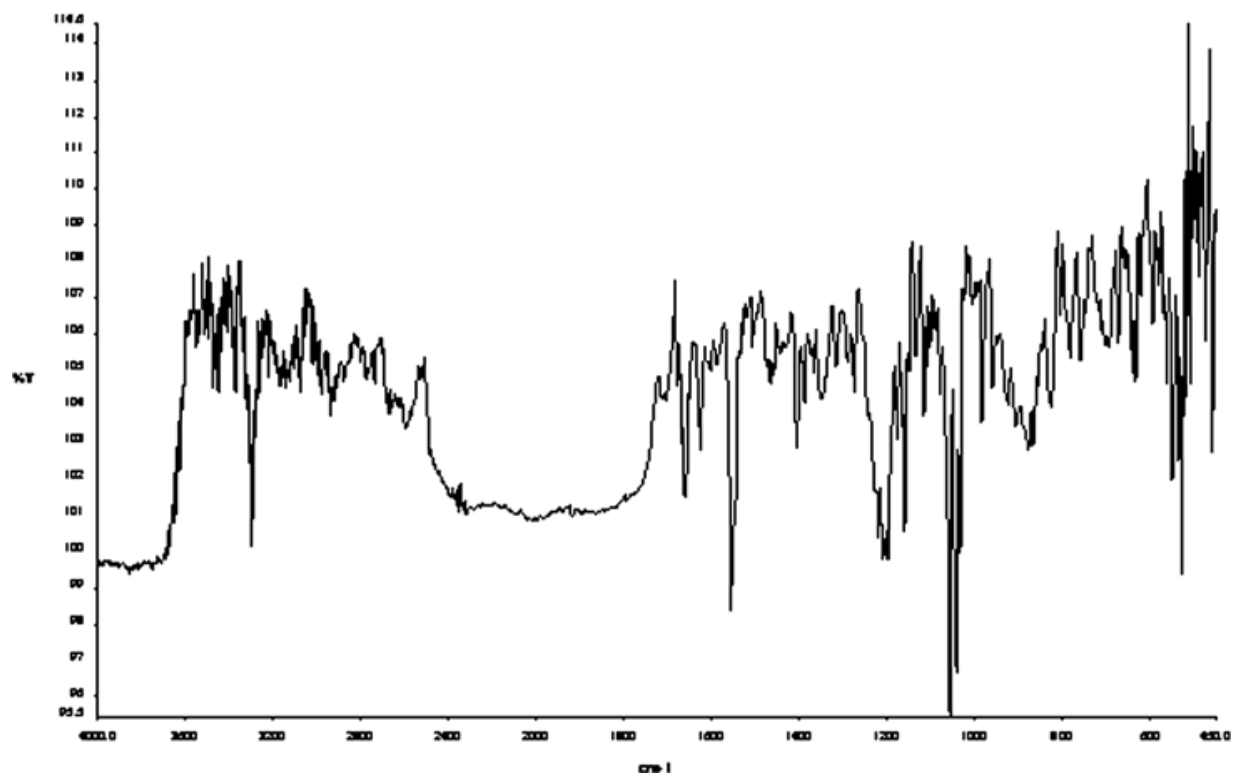

Figure 2. FT-IR characterization of AMPS - TEA. 


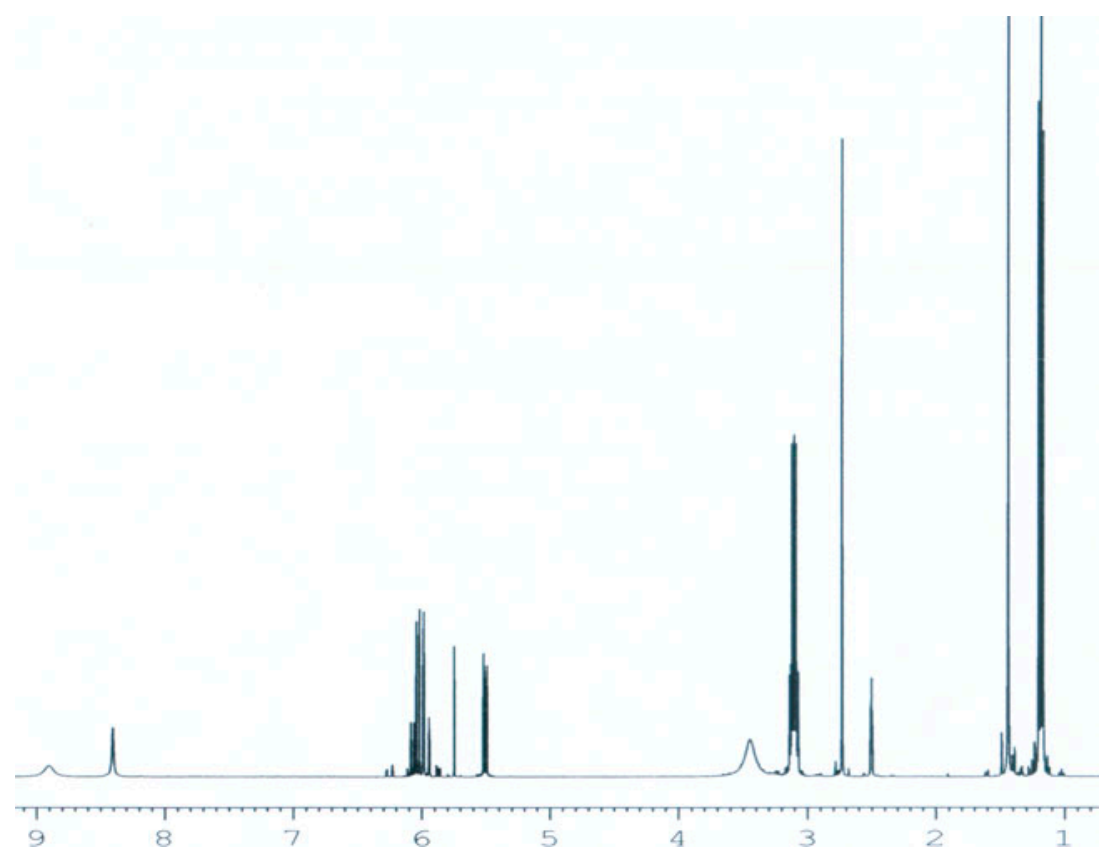

Figure 3. ${ }^{1} \mathrm{H}$ NMR characterization of AMPS - TEA.

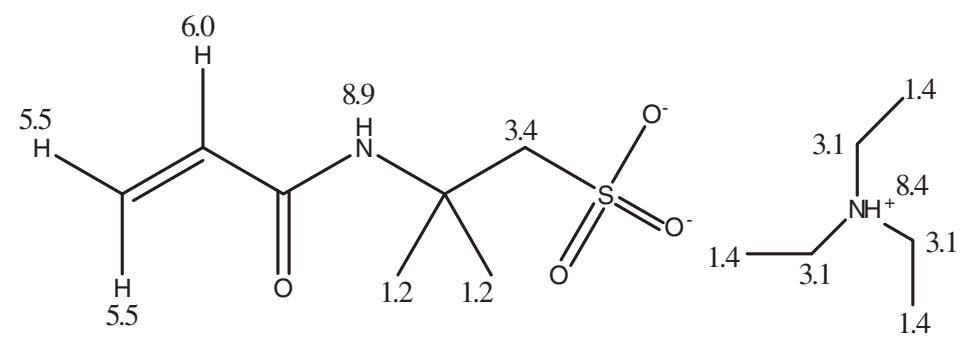

Figure 4. Structure of the resulted RTIL deducted from FT-IR and ${ }^{1} \mathrm{H}$ NMR.

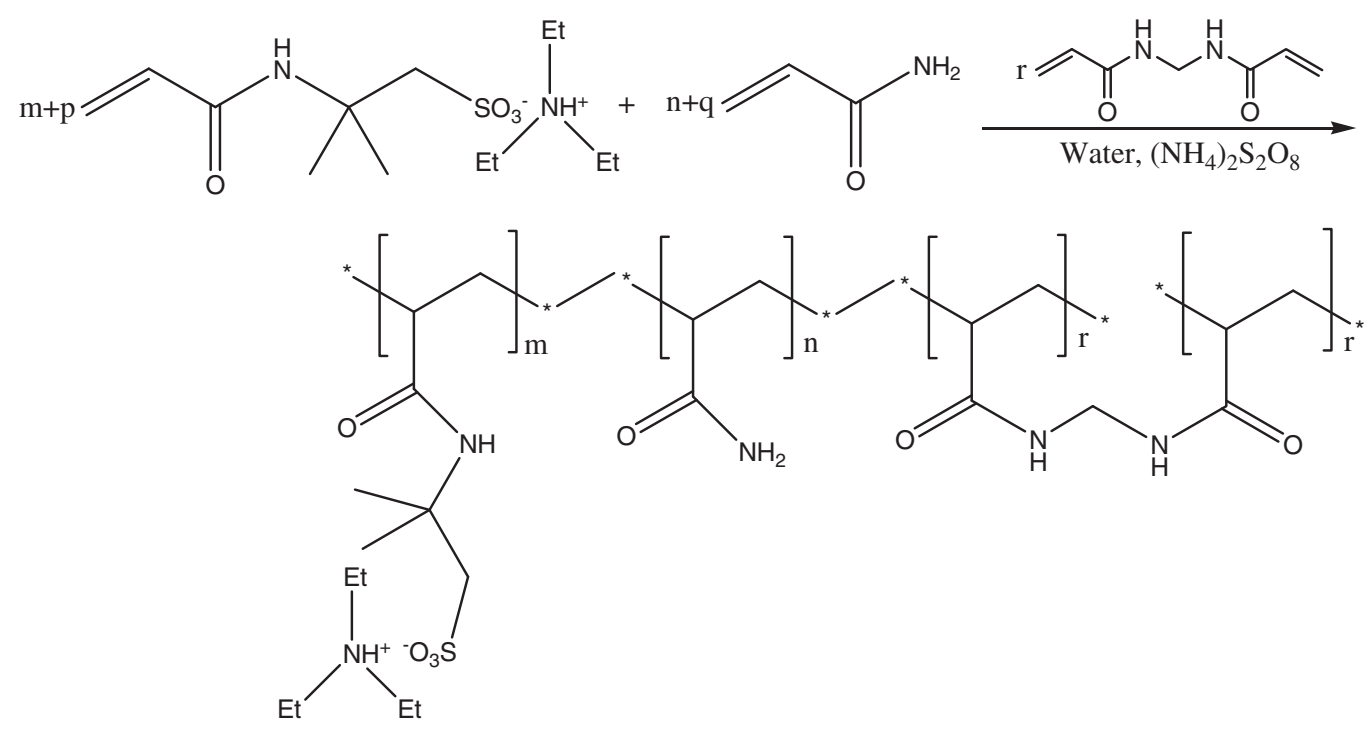

Scheme 3. Synthesis of poly(AMPS - TEA-co-AAm) gels. 


\section{Swelling of poly(AMPS - TEA-co-AAm) gels in organic solvents}

The swelling characteristic of poly(AMPS - TEA-co-AAm) gels in water and a variety of organic solvents was investigated. It showed poly(AMPS - TEA-co-AAm) gels can not only swell in water, but also in a variety of polar and nonpolar organic solvents. The superabsorbency of the poly (AMPS - TEA-co-AAm) gels with the composition of AAm $30 \%$, AMPS $70 \%$, and MBAm $0.20 \%$ (all of them calculated by the gross weight of AAm and AMPS) was investigated. A series of most conventional alcohols, chlorinated methanes, amines, and acetone, acetoneitril, dimethylsulfoxide were examined because these organic solvents are of significant importance commercially in wide varieties of applications. Surveying and studying new materials to highly imbibe these organic solvents will be of great potential importance. The dielectric constant (c) and the absorbency by the poly(AMPS - TEA-coAAm) gels of these solvents are shown in Table 1.

It was found that the poly(AMPS - TEA-co-AAm) gels showed superabsorbency for both water and a variety of organic solvents, irrespective of their polarity (dielectric constant). It has been widely known that super absorbent polymers swell and absorb water up to several hundred times their dried weights and have become ubiquitous and indispensable materials in many applications - their high swelling abilities in water originate from the electrostatic repulsion between the ions fixed on the polymer chains and osmotic pressure induced by freely mobile counterions in the networks of the

Table 1. The dielectric constant $(\varepsilon)$ and the absorbency by the poly(AMPS - TEA-co-AAm) gels of the solvents.

\begin{tabular}{lcc}
\hline Solvent & $\begin{array}{c}\text { Dielectric constant } \\
(\varepsilon)\end{array}$ & $\begin{array}{c}\text { Absorbency } \\
(\mathrm{g} / \mathrm{g})\end{array}$ \\
\hline Hexane & 1.8865 & 0 (Collapse) \\
Carbon tetrachloride & 2.24 & 0 (Collapse) \\
Butylamine & 4.71 & 35 \\
Chloroform & 4.81 & 0 (Collapse) \\
Dichloromethane & 8.93 & 0 (Collapse) \\
Tert-butanol & 12.47 & 23 \\
1-Butanol & 17.8 & 37 \\
2-Propanol & 20.18 & 38 \\
Acetone & 20.7 & 0 (Collapse) \\
1-Propanol & 20.8 & 40 \\
Ethanol & 24.5 & 51 \\
1,2-Propanediol & 27.5 & 38 \\
1-Methyl-2- & 32.55 & 42 \\
pyrrolidone & & 76 \\
Methanol & 32.7 & 35 \\
1,3-Propanediol & 35.1 & 0 (Collapse) \\
Acetoneitrile & 37.5 & 50 \\
Ethylene glycol & 41.4 & 41 \\
Glycerol & 46.53 & 68 \\
Dimethylsulfoxide & 46.7 & 218 \\
Water & 80.1 & \\
\hline
\end{tabular}

polyelectrolyte gels - charged polymers with associated counterions, while such polyelectrolyte gels hardly swell in organic liquids as a result of aggregation of ion pairs. [35-39] In this case, dissociation ability of the ionic partners on the polymer chains plays an important role during swelling of polyelectrolyte gels in a solvent. Rationalized by this principle, the incorporation of polymerizable RTILs that are melting salts at room temperature completely composed of discrete cations and anions into the chains of polyelectrolyte gels ought to inhibit aggregation of the ionic partners both in water and in organic liquids, which in turn would allow RTILs-based gels to behave as superabsorbent gels irrespective of their liquid environment.

In addition, it has been discussed that the swelling of a crosslinked neutral polymer without any charges on the backbones in a solvent is caused by the imbalance between the repulsive forces among the polymer chains and the contractile forces due to stretching of elastically active networked structures.[40,41] Thus, both the compatibility of the polymer chains with the solvents and the crosslinking density of the networked structures play a key role in the swelling and collapsing of neutral polymer gels. In the same way, for polyelectrolyte gels, poor solvent compatibility with the backbones will not allow penetration of the solvent molecules into the polymer networks, while poor solvent compatibility with the counterions will not allow the dissociation of the ionic partners. As a result, the polymer gels collapses, even though the ion pairs would be easily dissociated. Alexander-Katz and Leibler [42] have been theoretically discussing the effect of counterion solubility, instead of backbone solubility, on the stability of polyelectrolyte solutions. Therefore, it was thought that the solubility of counterion as well as the backbone of poly(AMPS TEA-co-AAm) gels resulted in the superabsorbency for both water and some organic solvents.

\section{Influence of AAm content in the gels on the swelling characteristic}

Various poly(AMPS - TEA-co-AAm) gels with different compositions were prepared by changing MBAm and AAm feeding during preparation of the reaction solution. It was found that the superabsorbency of poly(AMPS TEA-co-AAm) gels were seriously influenced by the composition. The effect of AAm feeding on the superabsorbency is shown in Figure 5. It was found that without AAm feeding, crosslinked homo-polymer of AMPS TEA was not transparent and glassy gel but muddy mixture without any strength, it did not absorb any solvent. Crosslinked poly(AMPS - TEA-co-AAm) also did not absorb any solvent as the AAm feeding was less than $15 \%$ (calculated by the gross weight of AMPS and AAm). Increased AAm feeding in the range of $15-30 \mathrm{wt} . \%$ (for 


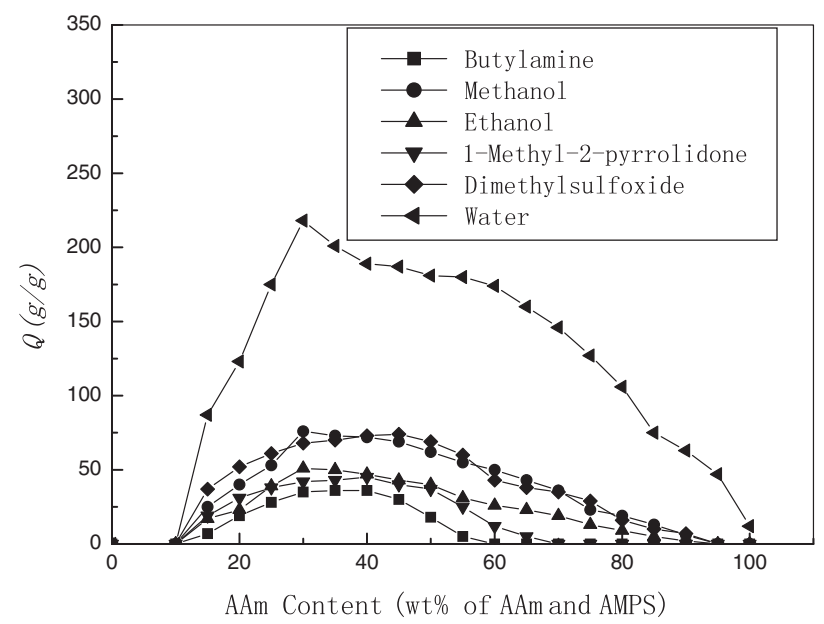

Figure 5. The effect of AAm (a) feeding on the absorbency.

water, methanol, and ethanol), $40 \mathrm{wt} \%$ (for butylamine and 1-methyl-2-pyrrolidone) or $45 \mathrm{wt} . \%$ (for dimethylsulfoxide), the absorbency of the gels increased accordingly; while the absorbency of the gels decreased as the AAm feeding continued to increase. The gels did not swell any more as the AAm feeding was more than $60 \mathrm{wt} . \%$ (for butylamine), $70 \mathrm{wt} . \%$ (for 1-methyl-2-pyrrolidone), $90 \mathrm{wt} \%$ (for dimethylsulfoxide), or $95 \mathrm{wt} . \%$ (for methanol and ethanol).

\section{Influence of MBAm content in the gels on the swelling characteristic}

The effect of MBAm feeding on the superabsorbency is shown in Figure 6. In the range of $0.05-0.20 \mathrm{wt} . \%$, the absorbency to water, methanol, and ethanol of poly (AMPS - TEA-co-AAm) gels increased with the increase in the dosage of MBAm, while the absorbency decreased with the increase in the dosage of MBAm in

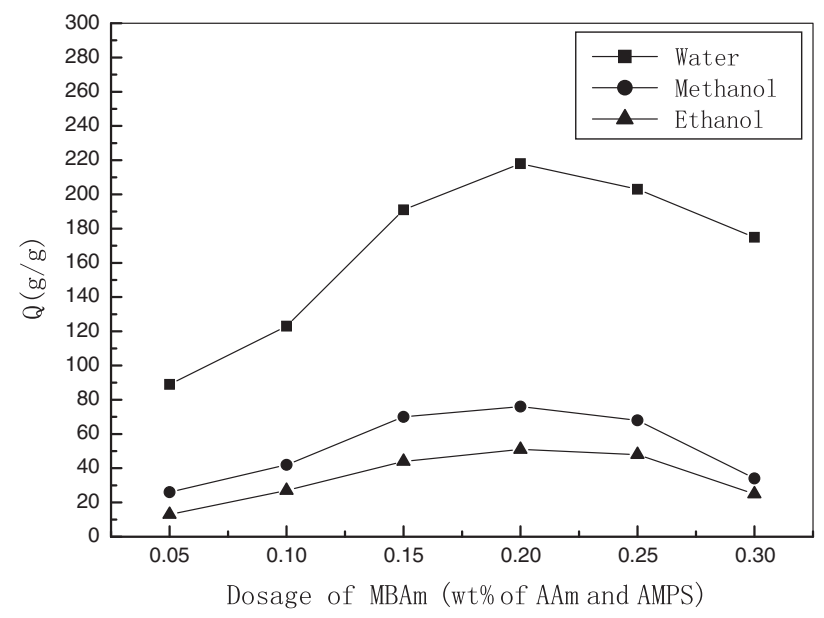

Figure 6. The effect of MBAm feeding on the absorbency. the range of $0.20-0.30 \mathrm{wt} . \%$. It was clear that a higher MBAm feeding induced an increased crosslinking density which resulted in a decreased absorbency; reducing the amount of crosslinker increased the swelling ability, but stable networks cannot form if the crosslinking density was too low.

\section{Conclusion}

A polymerizable RTIL, AMPS - TEA, was synthesis and characterized. DSC showed that the glass transition temperature of AMPS - TEA was about $-59.4^{\circ} \mathrm{C}$. Co-polymeric gels of AMPS - TEA with AAm were synthesized. Poly(AMPS - TEA-co-AAm) gels were transparent and glassy. The gels can swell in both water and a variety of organic solvents, irrespective of their polarity. The swelling mechanism of poly(AMPS TEA-co-AAm) gels lies in the dissociation ability of the ionic partners on the polymer chains as a result of incorporation of AMPS - TEA which is completely composed of discrete cations and anions into main chains of the polyelectrolyte gels, as well as the solubility of both counterion and the backbone in the solvents.

\section{Funding}

This work was supported by EU FP7 IRSES project 'ABREM' [grant agreement PIRSES-GA-2009-247599]; Dongguan Municipal Science \& Technology Planning Project of China [contract grant no. 201110810100327].

\section{References}

[1] Huddleston JG, Willauer HD, Swatloski RP, Visser AE, Rogers RD. Room temperature ionic liquids as novel media for 'clean' liquid-liquid extraction. Chem. Commun. 1998;16:1765-1766.

[2] Wilkes JS. Properties of ionic liquid solvents for catalysis. J. Mol. Catal. A: Chem. 2004;214:11-17.

[3] Fuller J, Carlin RT, Osteryoung RA. The room temperature ionic liquid 1-ethyl-methylimidazolium tetrafluoroborate: electrochemical couples and physical properties. J. Electrochem. Soc. 1997;144:3881-3886.

[4] Zhu HP, Yang F, Tang J, He MY. Brønsted acidic ionic liquid 1-methylimidazolium tetrafluoroborate: a green catalyst and recyclable medium for esterification. Green Chem. 2003;5:38-39.

[5] Carda-Broch S, Berthod A, Armstrong DW. Solvent properties of the 1-butyl-3-methylimidazolium hexafluorophosphate ionic liquid. Anal. Bioanal. Chem. 2003;375:191-199.

[6] Wasserscheid P, van Hal R, Bösmann A. 1-n-Butyl3-methylimidazolium (bmim) octylsulfate - an even 'greener' ionic liquid. Green Chem. 2002;4:400-404.

[7] Fuller J, Breda AC, Carlin RT. Ionic liquid-polymer gel electrolytes from hydrophilic and hydrophobic ionic liquids. J. Electroanal. Chem. 1998;459:29-34.

[8] Ue M, Takeda M, Toriumi A, Kominato A, Hagiwara R, Ito Y. Application of low-viscosity ionic liquid to the electrolyte of double-layer capacitors. J. Electrochem. Soc. 2003;150:A499-A502. 
[9] Rogers RD, Seddon KR. Ionic liquids - solvents of the future? Science. 2003;302:792-793.

[10] Biedron T, Kubisa P. Atom transfer radical polymerization of acrylates in an ionic liquid: synthesis of block copolymers. J. Polym. Sci., Part A: Polym. Chem. 2002;40:2799-2809.

[11] Zhang Y, Chen X, Lan J, You J, Chen L. Synthesis and biological applications of imidazolium-based polymerized ionic liquid as a gene delivery vector. Chem. Biol. Drug Des. 2009;74:282-288.

[12] Weingartner H. Understanding ionic liquids at the molecular level: facts, problems, and controversies. Angew. Chem. Int. Ed. 2008;47:654-670.

[13] Wasserscheid P, Keim W. Ionic liquids - new 'solutions' for transition metal catalysis. Angew. Chem. Int. Ed. 2000;39:3772-3789.

[14] Parvulescu VI, Hardacre C. Catalysis in ionic liquids. Chem. Rev. 2007;107:2615-2665.

[15] Dupont J, de Souza RF, Suarez PAZ. Ionic liquid (molten salt) phase organometallic catalysis. Chem. Rev. 2002;102:3667-3692.

[16] Hough WL, Rogers RD. Ionic liquids then and now: from solvents to materials to active pharmaceutical ingredients. Bull. Chem. Soc. Jpn. 2007;80:2262-2269.

[17] Biedron T, Kubisa P. Ionic liquids as reaction media for polymerization processes: atom transfer radical polymerization (ATRP) of acrylates in ionic liquids. Polym. Int. 2003;52:1584-1588.

[18] Kimizuka N, Nakashima T. Spontaneous self-assembly of glycolipid bilayer membranes in sugar-philic ionic liquids and formation of ionogels. Langmuir. 2001;17: 6759-6761.

[19] Zhang H, Hong K, Mays JW. Synthesis of block copolymers of styrene and methyl methacrylate by conventional free radical polymerization in room temperature ionic liquids. Macromolecules. 2002;35:5738-5741.

[20] Welton T. Room-temperature ionic liquids. Solvents for synthesis and catalysis. Chem. Rev. 1999;99:2071-2084.

[21] Mallakpour S, Seyedjamali H. Ionic liquid as a green media for rapid synthesis of optically active organosoluble polyamides. Des. Monomers Polym. 2010;13:377-386.

[22] Catel Y, Degrange M, Pluart LL, Madec P-J, Pham T-N, Chen F, Cook WD. Synthesis, photopolymerization, and adhesive properties of new bisphosphonic acid monomers for dental application. J. Polym. Sci., Part A: Polym. Chem. 2009;47:5258-5271.

[23] Mallakpour S, Dinari M. High-speed microwave-promoted direct poly-amidation reactions of bulky chiral dicarboxylic acide with different aromatic diamines in imidazolium types ionic liquid as a reaction medium. Des. Monomers Polym. 2010;13:51-64.

[24] Shaplov AS, Lozinskaya EI, Losada R, Wandrey C, Zdvizhkov AT, Korlyukov AA, Lyssenko KA, Malyshkina IA, Vygodskii YS. Polymerization of the new doublecharged monomer bis-1,3(N, N, N-trimethylammonium dicyanamide)-2-propylmethacrylate and ionic conductivity of the novel polyelectrolytes. Polym. Adv. Technol. 2011;22:448-457.

[25] Laschewsky A. Recent trends in the synthesis of polyelec trolytes. Curr. Opin. Colloid Interface Sci. 2012;17:56-63.

[26] Le Bideau J, Viau L, Vioux A. Ionogels, ionic liquid based hybrid materials. Chem. Soc. Rev. 2011;40:907-925.

[27] Armand M, Endres F, MacFarlane DR, Ohno H, Scrosati B. Ionic-liquid materials for the electrochemical challenges of the future. Nat. Mater. 2009;8:621-630.

[28] Mallakpour S, Khania M. Synthesis and characterization of poly(amide-imide)s bearing a S-valine moiety in molten ionic liquid. Des. Monomers Polym. 2011;14:221-232.

[29] Hosseinzadeh F, Mahkam M, Galehassadi M. Synthesis and characterization of ionic liquid functionalized polymers for drug delivery of an anti-inflammatory drug. Des. Monomers Polym. 2012;15:379-388.

[30] Ding S, Radosz M, Shen Y. Ionic liquid catalyst for biphasic atom transfer radical polymerization of methyl methacrylate. Macromolecules. 2005;38:5921-5928.

[31] Yuan J, Mecerreyes D, Antonietti M. Poly(ionic liquid)s: an update. Prog. Polym. Sci. 2013; Available from: http:// dx.doi.org/10.1016/j.progpolymsci.2013.04.002

[32] Yuan J, Antonietti M. Poly(ionic liquid)s: polymers expanding classical property profiles. Polymer 2011;52:1469-1482.

[33] Hirao M, Ito K, Ohno H. Preparation and polymerization of new organic molten salts: $\mathrm{N}$-alkylimidazolium salt derivatives. Electrochim. Acta. 2000;45:1291-1294.

[34] Ohno H. Molten salt type polymer electrolytes. Electrochim. Acta. 2001;46:1407-1411.

[35] Li X, Cui Y. Ultraviolet-induced decomposition of acrylic acid-based superabsorbent hydrogels crosslinked with N,N-methylenebisacrylamide. J. Appl. Polym. Sci. 2008; 108:3435-3441.

[36] Colby RH. Ionic partners split up. Nat. Mater. 2007;6:401-402.

[37] Li X, Pan X. Hydrogels based on hemicellulose and lignin from lignocellulose biorefinery: a mini-review. J. Bio-Based Mater. Bioenerg. 2010;4:289-297.

[38] Li X, Cui Y. Hydrogel-hydrogel composite the interfacial structure and interaction between water and polymer chains. J. Appl. Polym. Sci. 2008;108:3713-3719.

[39] Zhang B, Cui Y, Yin G, Li X. Adsorption of copper (II) and lead (II) ions onto cottonseed protein-PAA hydrogel composite. Polym.Plast. Technol. Eng. 2012;51:612-619.

[40] Ono T, Sugimoto T, Shinkai S, Sada K. Lipophilic polyelectrolyte gels as super-absorbent polymers for nonpolar organic solvents. Nat. Mater. 2007;6:429-432.

[41] Ono T, Shinkai S, Sada K. Discontinuous swelling behaviors of lipophilic polyelectrolyte gels in non-polar media. Soft Matter. 2008;4:748-750.

[42] Alexander-Katz A, Leibler L. Controlling polyelectrolyte equilibria and structure via counterion - solvent interactions. Soft Matter. 2009;5:2198-2207. 